\title{
A Review on Male Infertility - Environmental Factors, Pathophysiological and Oxidative Stress
}

\author{
Lata Kanyal Butola ${ }^{1}$, Archana Dhok², Deepika Kanyal' ${ }^{3}$, Anjali Vagga ${ }^{4}$ \\ 1, 2,4 Department of Biochemistry, Jawaharlal Nehru Medical College, Wardha, Maharashtra, India. \\ ${ }^{3}$ Department of Hospital Administration, Jawaharlal Nehru Medical College, Wardha, Maharashtra, India.
}

\section{ABSTRACT}

Male infertility is one of the rising global problems with an increasing decline in male semen quality among men living in Asia, Europe, Africa and North America. Infertility is defined as the failure of conception after at least 12 months of unprotected intercourse. Globally 70 million people are affected by infertility. Environmental, occupational and modifiable lifestyle factors may contribute to this decline of male fertility. Various factors associated with male infertility include smoking cigarettes, alcohol intake, use of illicit drugs, obesity, genetic factors, heavy metals, psychological stress, exposure to pesticides and industrial chemicals, poor nutrition intake, oxidative stress, sedentary lifestyle, advanced paternal age, diet and coffee consumption.

\section{KEY WORDS}

Infertility, Antioxidant, Environmental Factors, Endocrine Factors.
Corresponding Author: Dr. Lata Kanyal Butola, Department of Biochemistry, Jawaharlal Nehru Medical College, Wardha, Maharashtra, India.

E-mail: kanyallata1010@gmail.com

DOI: $10.14260 /$ jemds/2021/767

How to Cite This Article:

Butola LK, Dhok A, Kanyal D, et al. A review on male infertility - environmental factors, pathophysiological and oxidative stress. J Evolution Med Dent Sci 2021;10(44):3798-3804, 10.14260/jemds/2021/767

Submission 09-09-2021,

Peer Review 05-10-2021,

Acceptance 11-10-2021,

Published 30-11-2021.

Copyright (C) 2021 Lata Kanyal Butola et al. This is an open access article distributed under Creative Commons Attribution License [Attribution 4.0 International (CC $B Y 4.0)]$ 


\section{BACKGROUND}

Infertility is a global medical and social issue. Although infertility results from a male partner's abnormality in nearly 50 percent of cases, considering infertility as a problem for female partners is a common prejudice. In many cases, the underlying pathological basis of qualitative and quantitative sperm defects which lead to fertilization defects is not clear. Infertility is a major clinical issue that medically and psychosocially affects people. Infertility is defined as the inability to conceive after at least 12 months of unprotected intercourse. In the United States, about 15 percent of couples suffer from infertility. In India, it has been reported that one in six couples suffer from infertility. ${ }^{1-3}$

Numerous factors can impact a couple's fertility. The factors responsible for male infertility are, however, conveniently classified into pre-testicular, testicular and posttesticular factors. ${ }^{4,5}$ The pre-testicular factors are related to hormonal imbalance and poor general health, and the important pre-testicular causes are hypogonadism, drugs, alcohol, smoking, psychological stress, genetic abnormalities, chemotherapy and various types of medication such as anabolic steroids, cimetidine, spironolactone, phenytoin, sulfasalazine and nitrofurantoin. On the other hand, the testicular factors refer to conditions where despite hormonal support, the testes produce semen of low and poor quality. Age, chromosomal abnormality, seminoma, idiopathic oligospermia, varicocele, hydrocele and mumps are among the testicular causes of male infertility. ${ }^{6}$ Post-testicular factors decrease male fertility by affecting the male genital system after testicular sperm production and include genital tract defects as well as ejaculation problems. Some important post-testicular factors are blockage or absence of vas deferens, prostatitis, antisperm antibodies, retrograde ejaculation, obstruction of the ejaculatory duct, hypospadias and impotence. Even so, in several cases, it is quite difficult to identify the exact factor responsible for male infertility and the mechanism of the underlying poor semen quality deficiencies remain obscure. Consequently, potential nonconventional factors have been sought for the pathogenesis of male infertility, and recent studies have suggested that oxidative stress may have a profound role in male infertility. ${ }^{7}$

\section{Spermiogenesis}

Spermiogenesis is a complex process involving many changes in morphology, physiology and biochemistry. These changes include nuclear condensation, nucleus shaping, acrosome formation, cytoplasm elimination, development of a flagellum, and mitochondrial arrangement into the middle sperm piece. The nucleus contains decondensed chromatin at the beginning of spermiogenesis and is believed to be active transcription. But the nucleus replaces lysine and histidinerich histones with a series of basic proteins during the later stages of spermiogenesis. These are initially transitional proteins, and ultimately basic protamines rich in arginine and cysteine the spermatids becomes highly condensed. Replacement of nuclear histones during spermiogenesis by protamines and nuclear condensation involves a series of interactions mediated by the transition proteins.

Two major classes of transitional proteins TP1 and TP2 are found. ${ }^{8}$ As a result of this arrangement, mammalian sperm nuclear DNA becomes 6 times more condensed than somatic cell DNA (in an ordered process starting at the anterior end of the nucleus and proceeding towards the tail, making sperm nuclear DNA known as the highest condensed eukaryotic DNA). ${ }^{9}$ The formation of disulphide cross-links in the nucleus is an important change to the sperm during epididymal transit. Protamine, rich in cysteine, contains groups of sulfhydryl (S-H) that participate in the formation of covalent bonds in and between the molecules of protamine that provide a highly stable keratin nature. ${ }^{10}$ Human nuclear proteins at the end of the epididymal passage contain a limited amount of nonoxidized protamine and cysteine. These free amino groups of cysteine and arginine and free thiol may be chelated by reversible binding of zinc ions and further condensing of the sperm nucleus. Zinc in the sperm nucleus is thought to hinder the premature exchange of thiol disulphide and thereby decreases the vulnerability of sperm chromatin to chemical attack during transfer to the ovum. ${ }^{11}$ Until ejaculation, the storage of fully mature sperm occurs primarily in epididymis cauda. Sperm can remain viable in the epididymis for about 4 to 6 weeks. ${ }^{12}$ During this period, sperm may become exposed to toxicants. If such chemicals subvert the normal condensation mechanism to protect the nucleus, they may have a detrimental effect on the integrity and function of nuclear sperm. ${ }^{13}$

\section{CAUSES OF MALE INFERTILITY}

Environmental, occupational and modifiable factors in lifestyle may contribute to male infertility. Male infertility related lifestyle factors include cigarette smoking, alcohol intake, illicit drug use, obesity, psychological stress, advanced paternal age, diet and coffee consumption. Testicular heat stress, intense cycling training, insomnia and exposure to electromagnetic radiation from mobile phones are other factors associated with male infertility. ${ }^{14}$

\section{Cigarette Smoking}

Cigarette smoke exposure generates high oxidative stress levels, directly increasing both seminal leukocyte concentration and seminal ROS generation, ${ }^{15}$ and decreasing seminal levels of the antioxidant enzyme SOD. Men who smoke also have lower sperm quality measures including reduced sperm counts, motility and morphologically normal sperm counts. Smoking has been noted to decrease the concentration of seminal antioxidants vitamin $\mathrm{C}$ and $\mathrm{E}$, thereby reducing the spermatozoa and seminal fluid's oxidant scavenging capacity. ${ }^{16}$
Alcohol Intake
Alcohol can affect male infertility by altering sperm count, shape, motility and size of sperm. Heavy drinking of alcohol affects fertility by lowering testosterone levels, follicle- stimulating hormones and luteinizing hormones and raising estrogen levels, which reduce sperm production. It has also been shown that high amounts of alcohol intake increase systemic levels of oxidative stress, and the effect of oxidative stress can be further exacerbated by the low-nutrient diet that usually accompanies this high intake of alcohol. ${ }^{17}$ 


\section{Industrial Compounds}

Industrial waste products have a broad impact on human and environmental health and several groups have been investigating the impact of these compounds on fertility. The compound phthalate, for example, is found in a wide range of plastics used in food packaging, as well as beauty products and exposure to these chemicals can occur via oral, skin, or inhalation routes. Exposure to phthalates has been shown to cause sperm DNA damage, as well as impaired spermatogenesis. ${ }^{18,19}$ Heavy metals such as cadmium and lead, have been shown to increase testicular oxidative stress and increased infertility and miscarriage rates have been noted in welders as well as paint workers, and battery manufacturers. ${ }^{20}$ Men in these industries may be exposed to heavy metals and are recommended to take aggressive steps to avoid further exposure. Several pesticides, including lindane, methoxychlor and herbicide dioxin-TCDD, have been linked to increased testicular oxidative stress in rodent models. It has been noted that the almost ubiquitous food preservative sulphur dioxide directly increases intratesticular oxidative stress, and it has been shown that diesel exhaust particulates powerfully activate the production of leukocyte ROS. 21,22

\section{Male Infertility}

\section{Impairment of Spermatozoa in Humans}

Defective sperms are one of the main causes of infertility. It has been reported that the nucleohistone sperm compartment containing histone bound DNA sequences such as telomeres and gene promoters for embryonic development is often at high risk of oxidative stress, leading to infertility. ${ }^{23}$ The relative decrease in protamine 2 levels also results in infertility in the ratio of protamine 1: protamine 2, at mRNA and protein rates. Once, gene mutations encoding protamines induce structural changes in the composition of the sperm chromatin, leading to infertility. ${ }^{24}$

\section{Sperm Dysfunction}

The mechanism of sperm dysfunction is related to oxidative stress, which induces distortion of sperm DNA integrity by destroying the lipids and protein present in the sperm cells. This has a negative effect on sperm cell membrane fluidity and permeability which results in infertility. ${ }^{25}$

\section{Sperm DNA Fragmentation (SDF)}

Many researchers say that SDF is regulated by free radicals. ${ }^{26}$ Once again, the underlying SDF mechanism includes single and double-strand breaks, DNA fragmentation, abasic site entry, purine, pyrimidine modifications and DNA cross-linking resulting in gene transcription induction, signal transduction pathways induction; accelerated telomeric RNA depletion, replication defects, genomic instability, and GC to TA transmissions. ${ }^{27}$ Given that these mechanisms are also known to be the causes of carcinogenesis, this may explain a link between infertility and cancer. Male infertility is also closely associated with DNA damage caused by ROS, which in turn accelerates the apoptosis of the germ cells, leading to a decrease in sperm count. ${ }^{28}$

\section{Physiologic Role of Reactive Oxygen Species (ROS)}

Depending on the type and concentration of ROS, and the length and location of ROS exposure, it has adverse effects on sperm function. ${ }^{29}$ Sperm gradually acquires the capacity to travel during epididymal motion. However, they gain the capacity to fertilize in the female tract by a series of physiological changes, called "Capacitation". 30

\section{Antioxidants and Infertility}

ROS has both physiological and pathological functions, and antioxidants maintain a stable state of ROS in seminal plasma. Antioxidants serve as free radical scavengers, to shield ROS sperm. Such antioxidants are superoxide dismutase (SOD), catalase and glutathione peroxidase (GPX). However, semen contains a variety of non-enzymatic antioxidants such as Vitamin C and E, pyruvate, glutathione and carnitine, which decrease endogenous mechanism of repair and enzymatic defence. ${ }^{31}$ The production of reactive oxygen species may result in the breakage of the DNA strands and chromosomal rearrangements. $8-0 \mathrm{HdG}$ is a sensitive marker of oxidative stress, many studies have shown a higher level of 8-OHdG in ejaculated sperm of infertile males.

\section{Seminal Malondialdehyde}

Lipid peroxidation of the sperm membrane is the main mechanism of ROS-induced sperm damage that leads to infertility. Malondialdehyde, a by-product of lipid peroxide, reflects the level of lipid peroxidation. ${ }^{32}$ Numerous studies have shown that lipid peroxidation affects sperm concentration, motility, morphology and poor sperm quality.

\section{Glutathione}

Glutathione is one of the most abundant non-thiol proteins in mammalian cells. ${ }^{33}$ Glutathione deficiency can lead to midpiece instability, leading to deficient motility. ${ }^{34}$ It protects the plasma membrane from lipid peroxidation, and superoxide prevents the formation of 02. Glutathione peroxidase (GPx) is a major contributor to the reduction of hydrogen peroxide and organic peroxide involving phospholipids peroxides. Selenium occurs in the GPx active site in the selenocysteine. It is contained in the mitochondrial sperm matrix but a nuclear portion of GPx is associated with the protection of sperm DNA from oxidative disruption. The nuclear process has also been documented to play a part in condensing chromatin. The appearance of GPx in seminal plasma indicates that its source may be the prostate. ${ }^{35}$

\section{Superoxide Dismutase}

Superoxide dismutase (SOD) scavengers both extracellular and intracellular superoxide anion and prevent the lipid peroxidation of the plasma membrane. ${ }^{36}$ SOD also prevents the premature hyperactivation and pre-ejaculation capacitation of superoxide radicals. ${ }^{37}$ Superoxide dismutase or superoxide reductase catalyzes the conversion of superoxide anions to dismutation. These also occur for both extra- and intracellular forms which are otherwise known as metalloenzymes. 
The two intracellular forms in their active centre and the organelle where they are located are separated by the metal. The first intracellular component in the active centre contains copper and zinc (SOD-1) and is mainly contained in the cytoplasm, while the second component present with manganese in the active centre of mitochondria is called SOD2. In extracellular space (SOD-3), the SOD functions as an extracellular shape. This is connected to the surface of polysaccharides although it can be found in a free form. ${ }^{38} \mathrm{SOD}$ is one of the key elements of seminal plasma. Superoxide anion scavenging capacity and several investigators have reported a reduction of SOD activity in the semen of infertile males.

\section{Catalase}

Catalase catalyzes the splitting of $\mathrm{H} 202$ into $\mathrm{H} 2 \mathrm{O}$ and 02 ; $\mathrm{H} 202$ is one of the reactive oxygen species involved in oxidative stress; catalase helps detoxify both intracellular and extracellular $\mathrm{H} 2 \mathrm{O} 2$ into water and oxygen. However, it triggers nitrous oxide (NO) sperm capacitation, a complex process involving $\mathrm{H} 2 \mathrm{O} 2 .{ }^{39}$

\section{Vitamin E}

Vitamin E is a powerful antioxidant that is found in the cell membrane. It inhibits lipid peroxidation and scavenges free radicals produced during univalent molecular oxygen reduction and normal oxidative activity. The formation of these radicals leads to phospholipid peroxidation in the mitochondrial sperm resulting in low motility ${ }^{40}$ Suleiman et al. (1996) found that supplementation with vitamin E could significantly reduce lipid peroxidation in seminal plasma, improve sperm motility and improve the incidence of pregnancy. 41

\section{Vitamin C}

Vitamin $\mathrm{C}$ is a keto-lactone containing six carbons that are biosynthesized in the liver. Nevertheless, human inability to synthesize vitamins necessitates its inclusion in the diet or as a supplement. Vitamin C is a cofactor enzyme, which helps in the metabolic folic acid, tyrosine, and tryptophane processes. 42

\section{Endocrine Factors of Male Infertility}

\section{Hypothalamic Dysfunction (Kallmann} Syndrome)

The normal endocrine reproductive system functions as a prerequisite for normal male fertility. Any disruption of the delicately coordinated interaction between the hypothalamic-pituitary-testicular axis components may result in hypogonadism or infertility. The objective of the clinical evaluation is to determine whether the patient has an abnormality and whether hormone therapy will correct infertility. Based on a careful history, the physician will determine whether the patient's hypogonadism is (1) prepubertal or postpubertal onset; (2) the result of an abnormality in the hypothalamicpituitary axis, the tests or the androgen receptor; or (3) the result of another underlying medical condition. This information will place the patients in one of the four diagnostic categories: hypogonadotropic hypogonadism, testicular failure, deficiency of $5 \alpha$ - reductase, or resistance to androgen disorders with identifiable pathogenic mechanism fall within each category. 43

\section{Pituitary Failure (Tumour, Radiation,} Surgery)

The failure of the pituitary to secrete the folliclestimulating hormone and luteinizing hormone results in testicular disruption and infertility. Measurement of serum FSH is of paramount importance, providing a useful index of the seminiferous epithelium state when the concentration is related to sperm density: high concentration associated with severe oligospermia or azoospermia usually denotes untreatable infertility. In about $30 \%$ of men with severe degrees of testicular damage, elevated LH and low testosterone levels are indicative of interstitial cell failure. Measurements of prolactin are most likely linked to impotence rather than infertility. Hormonal treatment is often indicated for male infertility. Androgen injections of testosterone suppress spermatogenesis, so sperm count will rebound to a concentration greater than pretreatment levels once treatment is stopped. Those endocrine factors can potentiate testicular damage and are postulated as indicators of seminiferous tubular disruption resulting in spermatogenesis disruption, based on FSH measurement. ${ }^{4}$

\section{Hyperprolactinemia (Drug, Tumour)}

Hyperprolactinemia causes infertility in around $11 \%$ of oligospermic males. Hyperprolactinemia inhibits the pulsatile secretion of the gonadotrophin-releasing hormone, which causes the decreased pulsatile release of follicle-stimulating hormone, luteinizing hormone, and testosterone, which in turn causes spermatogenic arrest, impaired sperm motility, and altered sperm quality. It later produces secondary hypogonadism and infertility. Hyperprolactinemia also directly influences spermatogenesis and steroidogenesis by acting on prolactin receptors present in Sertoli cells and Leydig cells in testes and produces primary hypogonadism and infertility. It is seen that oligospermia or azoospermic patients with normal serum levels of gonadotrophins show relatively higher serum levels of prolactin, proving a role of prolactin in gametogenesis, which is independent of gonadotrophins. Many studies are suggesting that hyperprolactinemia has a definite role in male infertility, and is one of the reversible causes of infertility. It can be managed medically with simple medication, such as bromocriptine and cabergoline, which normalize serum prolactin levels, restoration of gonadal function, reversing infertility caused by hyperprolactinemia and induce a reduction in the prolactinoma size in the majority of patients. ${ }^{45}$

\section{Exogenous Androgens}

Exogenous androgens should only be prescribed if hypogonadism has been established by appropriate investigation, and preferably the patient does not intend to father a child. There are alternative 
medications or combinations of medications, that can be used if hypogonadism is present and fertility is desired. It is somewhat counterintuitive that testosterone treatment will decrease or abolish fertility. Exogenous testosterone inhibits spermatogenesis by removing the feedback response to low testosterone at the hypothalamus and pituitary. This results in reduced synthesis and secretion of gonadotropins required to stimulate endogenous testosterone production and to support spermatogenesis. It is important to realize that the normal testicular levels of testosterone are approximately 100 times the concentration in circulation. These high levels are required locally to support spermatogenesis. So even with circulating androgen levels within the normal range, spermatogenesis fails due to insufficient gonadotropin and local testosterone support. Androgenic herbal supplements and illicit use of anabolic steroids have contributed to this serious challenge in the treatment of infertile men. Most men will recover normal spermatogenesis after cessation of exogenous testosterone treatment, but this requires 6 months or more in most men. In rare cases, fertility is permanently impaired. 46

\section{Thyroid Disorders}

Thyroid dysfunction can impair semen quality, and decrease sperm motility and/or concentration. In addition, it is described as an association between TAI and asthenozoospermia. After patients achieved euthyroidism, alterations in semen were reversible. The impact of thyroid dysfunction on the semen is primarily due to an effect on the central (luteinizing hormone / follicle-stimulating hormone) and peripheral gonadal function and binding proteins via sex hormone. ${ }^{47}$

\section{Adrenal Hyperplasia}

The presence of testicular adrenal rest tumours (TARTs) in adult patients with congenital adrenal hyperplasia (CAH) is an important cause of gonadal dysfunction and infertility. 48

\section{Testicular Failure}

Testicular or spermatogenic insufficiency is the most serious form of male infertility. Typical testicular failure phenotype in severely impaired spermatogenesis results in azoospermia or severe oligozoospermia (sperm density as low as 5 or $106 \mathrm{ml}-1$ ). A range of conditions can cause testicular failure, both congenital and acquired. Even after a complete diagnostic workup, aetiology remains unknown in about 40-50 percent of primary testicular failure. ${ }^{49}$

\section{Anatomic}

1. Congenital absence of vas deferens.

2. Obstructed vas deferens.

3. Congenital abnormalities of the ejaculatory system.

4. Varicocele.

5. Retrograde ejaculations.
Abnormal Spermatogenesis
1. Unexplained azoospermia.
2. Chromosomal abnormalities.
3. Mumps orchitis.
4. Cryptorchidism.
5. Chemical or radiation exposure.

\section{Abnormal Motility \\ 1. Absent cilia (Kartagener syndrome) \\ 2. Antibody formation.}

\section{Psychosocial}

1. Unexplained impotence.

2. Decreased libido. 50

\section{CONCLUSIONS}

Antioxidants such as vitamin $\mathrm{C}$, coenzyme $\mathrm{Q}$, vitamin $\mathrm{E}$ and glutathione have been reported to be beneficial in male infertility management. Several defence mechanisms are critical in the living system, including antioxidant enzymes, vitamins and biomolecules. In essence, normal spermatozoa function may require a balance between ROS and antioxidants. Although natural antioxidants and phytocompounds have been shown to play a beneficial role in spermatogenesis. Infertility can be stressful for you as well as your partner. There are so many complications of male infertility like surgery or other procedures to treat low sperm count underlying causes or other reproductive problems, costly, involving reproductive techniques, stress and relationship difficulties associated with the impossibility of having a child. Almost all kinds of male infertility can't be prevented. You can however avoid certain known causes of male infertility. Nonetheless, healthy living, regular exercise and stress-free jobs can help reverse sperm dysfunction. For instance, don't smoke, limit alcohol intake, keep off weight, do not have a vasectomy, avoid things that cause the testicles to get prolonged heat, cut back on stress and avoid exposure to pesticides, heavy metals and other toxins and a healthy diet rich of antioxidants should be taken for the reversal of damage caused by reactive oxygen species.

Financial or other competing interests: None.

Disclosure forms provided by the authors are available with the full text of this article at jemds.com.

\section{REFERENCES}

[1] Snick HK, Snick TS, Evers JL, et al. The spontaneous pregnancy prognosis in untreated subfertile couples: the Walcheren primary care study. Hum Reprod 1997;12(7):1582-8.

[2] Butola LK, Ambad RS, Mahakalkar C, ML, et al. Oxidative stress and anti oxidant status in male infertility. European Journal of Molecular \& Clinical Medicine 2021;8(1):340-8. 
[3] Butola LK, Ambad R, Vagga A. Recent updates in female infertility: a short review of literature. Indian Journal of Forensic Medicine \& Toxicology 2021;15(2):302-11.

[4] Cerilli LA, Kuang W, Rogers D. A practical approach to testicular biopsy interpretation for male infertility. Arch Pathol Lab Med 2010;134(8):1197-204.

[5] Un-Nahar Z, Ali M, Biswas SK, et al. Study of seminal MDA level as a oxidative stress marker in infertile male. Journal of Science Foundation 2011;9(1-2):85-93.

[6] Un-Nahar Z, Ali M, Biswas SK, et al. Study of seminal MDA level as a oxidative stress marker in infertile male. Journal of Science Foundation 2011;9(1-2):85-93.

[7] Khosrowbeygi A, Zarghami N. Levels of oxidative stress biomarkers in seminal plasma and their relationship with seminal parameters. BMC Clin Pathol 2007;7:6.

[8] O'Donnell L. Mechanisms of spermiogenesis and spermiation and how they are disturbed. Spermatogenesis 2014;4(2):e979623.

[9] Champroux A, Torres-Carreira J, Gharagozloo P, et al. Mammalian sperm nuclear organization: resiliencies and vulnerabilities. Basic Clin Androl 2016;26:17.

[10] Emelyanov AV, Fyodorov DV. Thioredoxin-dependent disulfide bond reduction is required for protamine eviction from sperm chromatin. Genes Dev 2016;30(24):2651-6.

[11] Bjorndahl L, Kvist U. Structure of chromatin in spermatozoa. Adv Exp Med Biol 2014;791:1-11.

[12] Bedford JM. The epididymis re-visited: a personal view. Asian J Androl 2015;17(5):693-8.

[13] Vidal JD, Whitney KM. Morphologic manifestations of testicular and epididymal toxicity. Spermatogenesis 2014;4(2):e979099.

[14] Durairajanayagam D. Lifestyle causes of male infertility. Arab J Urol 2018;16(1):10-20.

[15] Arabi M, Mosthagi H. Influence of cigarette smoking on spermatozoa via seminal plasma. Andrologia 2005;37(4):119-24.

[16] Vine MF. Smoking and male reproduction: a review. Int J Androl 1996;19(6):323-37.

[17] Koch OR, Pani G, Borrello S, et al. Oxidative stress and antioxidant defenses in ethanol-induced cell injury. Mol Aspects Med 2004;25(1-2):191-8.

[18] Agarwal DK, Maronpot RR, Lamb JC, et al. Adverse effects of butyl benzyl phthalate on the reproductive and hematopoietic systems of male rats. Toxicology 1985;35(3):189-206.

[19] Acharya UR, Acharya S, Mishra M. Lead acetate induced cytotoxicity in male germinal cells of Swiss mice. Ind Health 2003;41(3):291-4.

[20] Bonde JP. The risk of male subfecundity attributable to welding of metals. Studies of semen quality, infertility, fertility, adverse pregnancy outcomes and childhood malignancy. Int J Androl 1993;16 Suppl 1:1-29.

[21] Chitra KC, Sujatha R, Latchoumycandane C, et al. Effect of lindane on antioxidant enzymes in epididymis and epididymal sperm of adult rats. Asian J Androl 2001;3(3):205-8.

[22] Alaghmand M, Blough NV. Source-dependent variation in hydroxyl radical production by airborne particulate matter. Environ Sci Technol 2007;41(7):2364-70.

[23] Bisht S, Faiq M, Dada R, et al. Oxidative stress and male infertility. Nat Rev Urol 2017;14(8):470-85.
[24] Jiang W, Sun H, Zhang J, et al. Polymorphisms in protamine 1 and protamine 2 predict the risk of male infertility: a metaanalysis. Sci Rep 2015;5:15300.

[25] Aitken RJ. Human spermatozoa: revelations on the road to conception. F1000 Prime Rep 2013;5:39.

[26] Dorostghoal M, Kazeminejad SR, Shahbazian N, et al. Oxidative stress status and sperm DNA fragmentation in fertile and infertile men. Andrologia 2017;49(10).

[27] Bauer NC, Corbett AH, Doetsch PW. The current state of eukaryotic DNA base damage and repair. Nucleic Acids Res 2015;43(21):10083-101.

[28] Aitken RJ, Krausz C. Oxidative stress, DNA damage and the Y chromosome. Reproduction 2001;122(4):497-506.

[29] Agarwal A, Saleh RA. Role of oxidants in male infertility: rationale, significance, and treatment. Urol Clin North Am 2002;29(4):817-27.

[30] Visconti PE, Kopf GS. Regulation of protein phosphorylation during sperm capacitation. Biol Reprod 1998;59(1):1-6.

[31] Yang J, Liu X, Bhalla K, et al. Prevention of apoptosis by Bcl-2:release of cytochrome c from mitochondria blocked. Science 1997;275(5303):1129-32.

[32] Sikka SC. Oxidative stress and role of antioxidants in normal and abnormal sperm function. Front Biosci 1996;1:78-86.

[33] Irvine DS. Glutathione as a treatment for male infertility. Rev Reprod 1996;1(1):6-12.

[34] Ursini F, Heim S, Kiess M. Dual function of the selenoprotein PHGPx during sperm maturation. Science 1999;285(5432):1393-6.

[35] Lenzi A, Sgro P, Salacone P, et al. A placebo-controlled double-blind randomized trial of the use of combined lcarnitine and lacetyl-carnitine treatment in men with asthenozoospermia. Fertil Steril 2004;81(6):1578-84.

[36] Aitken RJ, Roman SD. Antioxidant systems and oxidative stress in the testes. Oxid Med Cell Longev 2008;1(1):1524.

[37] Jeulin C, Soufir JC, Weber P, et al. Catalase activity in human spermatozoa and seminal plasma. Gamete Res 1989;24(2):185-96.

[38] Fraczek M, Kurpisz M. The redox system in human semen and peroxidative damage of spermatozoa. Postepy Hig Med Dosw (Online) 2004;59:523-34.

[39] Baker HW, Brindle J, Irvine DS, et al. Protective effect of antioxidants on the impairment of sperm motility by activated polymorphonuclear leukocytes. Fertil Steril 1996;65(2):411-9.

[40] Lamirande E, Leclerc P, Gagnon C. Capacitation as a regulatory event that primes spermatozoa for the acrosome reaction and fertilization. Mol Hum Reprod 1997;3(3):175-94.

[41] Palamanda JR, Kehrer JP. Involvement of vitamin E and protein thiols in the inhibition of microsomal lipid peroxidation by glutathione. Lipids 1993;28(5):427-31.

[42] Subramanian VS, Marchant JS, Reidling JC, et al. NGlycosylation is required for $\mathrm{Na}$--dependent vitamin $\mathrm{C}$ transporter functionality. Biochem Biophys Res Commun 2008;374(1):123-27.

[43] Sokol RZ. Endocrinology of male infertility: evaluation and treatment. Semin Reprod Med 2009;27(2):149-58.

[44] De Kretser DM. Endocrinology of male infertility. Br Med Bull 1979;35(2):187-92. 
[45] Singh P, Singh M, Cugati G, et al. Hyperprolactinemia: an often missed cause of male infertility. J Hum Reprod Sci 2011;4(2):102-3.

[46] Drobnis EZ, Nangia AK. Exogenous androgens and male reproduction. Adv Exp Med Biol 2017;1034:25-8.

[47] Poppe K, Glinoer D, Tournaye H, et al. Is systematic screening for thyroid disorders indicated in subfertile men? Eur J Endocrinol 2006;154(3):363-6.

[48] Claahsen-Van der Grinten HL, Otten BJ, Stikkelbroeck $\mathrm{MM}$, et al. Testicular adrenal rest tumours in congenital adrenal hyperplasia. Best Pract Res Clin Endocrinol Metab 2009;23(2):209-20.

[49] Song SH, Chiba K, Ramasamy R, et al. Recent advances in the genetics of testicular failure. Asian J Androl 2016;18(3):350-5.

[50] Burtis CA, Ashwood ER, Bruns DE. Reproductive endocrinology and related disorders. In: Tietz fundamentals of clinical chemistry. $5^{\text {th }}$ edn. St Louis, MO: Elsevier 2014: p. 1968. 